\title{
DOENÇA FALCIFORME NA GRAVIDEZ
}

\section{SICKLE CELL DISEASE IN PREGNANCY}

\author{
Tayna Lima da Silva'; Railson Henneberg ${ }^{2}$; Paulo Henrique da Silva ${ }^{2}$ \\ 1 Farmacêutica , especialista em Análises Clinicas, Faculdade Cathedral//BRAS \\ 2 Professor Doutor, Departamento de Análises Clínicas, UFPR
}

\section{RESUMO:}

Dentre as doenças monogênicas, as síndromes falciformes são as mais comuns e frequentes na população brasileira. Aliado às complicações que estas síndromes causam aos indivíduos afetados, a gravidez é um fator complicador, tornando uma gestação nestas condições, uma situação de alto risco tanto para a mãe quanto para o feto. O presente trabalho trata-se de uma pesquisa bibliográfica com o objetivo de pesquisar as complicações mais ocorrentes em mulheres portadoras de anemia falciforme possuem durante o período gestacional. Através de pesquisa bibliográfica realizada as principais e mais frequentes complicações da gravidez nas gestantes com síndrome falciforme são o aumento da incidência de infecções urinárias, aumento das crises dolorosas, parto pré-termo, anemia, bolsa rota, complicações cardíacas, síndrome do tórax agudo e necessidade de intervenção cirúrgica para o parto, com consequente aumento da mortalidade materno fetal. Desta forma, fica evidente a necessidade de maiores cuidados com este tipo de paciente durante a gestação.

Palavras-chave: Anemia falciforme, hemoglobinopatias, complicações gestacionais.

\section{ABSTRACT:}

Among the monogenic diseases, sickle cell disease are the most common and frequent in the Brazilian population. Allied complications that cause these syndromes to affected individuals, pregnancy is a complicating factor making gestation in these conditions, high risk for both the mother and the fetus. This work is in a literature search with the aim of researching the most complications occurring in women with sickle cell anemia have during the period of gestacional.Através bibliographic survey the main and most frequent complications of pregnancy in women with sickle cell syndrome are the increased incidence of urinary tract infections, increased pain crises , preterm delivery, anemia , rupture of membranes, cardiac complications, acute chest syndrome and the need for surgical intervention for delivery, with consequent increased fetal and maternal mortality. Thus, there is an evident need for greater care with this type of patient during pregnancy.

Keywords: Sickle cell anemia, hemoglobinopathies, gestational complications. 


\section{INTRODUÇÃO}

As doenças falciformes constituem um grupo de doenças genéticas que tem como característica comum a presença da hemoglobina $S$, proteína mutante, cuja principal característica é a de sofrer polimerização sob baixa tensão de oxigênio. $A$ hemoglobina S, polimerizando-se dentro dos eritrócitos, deforma-os, fazendo com que os mesmos assumam a forma de foice (Figura 1). A anemia falciforme (SS), a doença da hemoglobina $C$ falciforme (SC) e a associação entre a doença falciforme e a talassemia $ß$ (talassemia $S-\beta$ ) são as mais comuns das síndromes falciformes (LEVENO et al., 2010; STUART;NAGEL,2004).

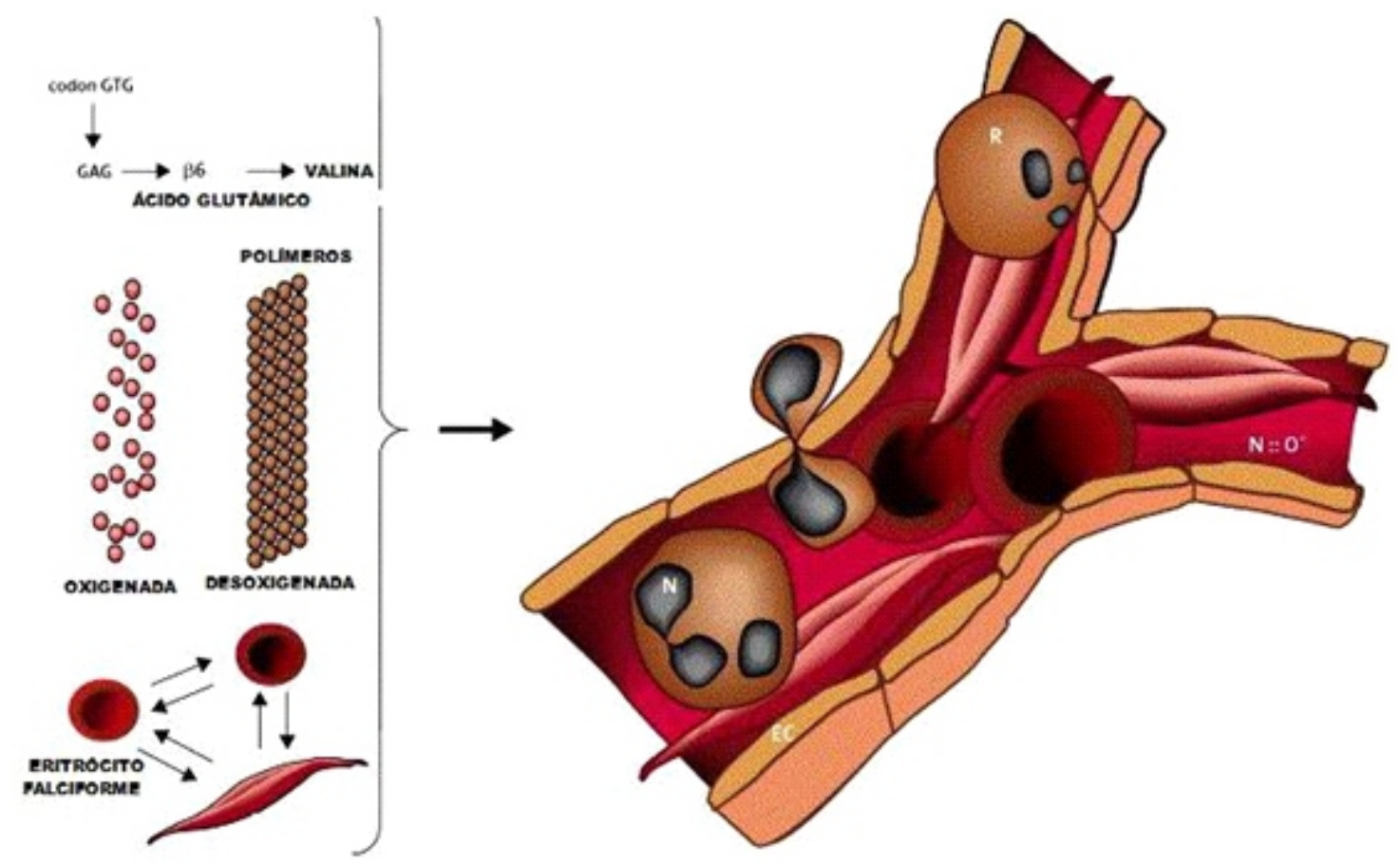

FIGURA1 - FISIOPATOLOGIADAVASOOCLUSÃO.

FONTE: (STUART;NAGEL,2004)

Descrita pela primeira vez por Herrick em 1910, a anemia falciforme é originária da África vindo para a América através do comércio de escravos, disseminando-se heterogeneamente pelo Brasil até metade do século XIX, sendo hoje a doença hereditária mais comum em nosso país (PERIN, 2002). Segundo Failace (2009) a alta frequência desta alteração molecular da hemoglobina se deva a séculos de seleção natural, pela maior resistência dos eritrócitos com Hb $\mathrm{S}$ à infecção pelo Plasmodium falciparum. A prevalência africana original diluiu-se pela miscigenação racial; passando a ser encontrada mesmo em pessoas aparentemente brancas.

Sendo uma das doenças hereditárias mais comuns no Brasil e no mundo, a 
anemia falciforme tem maior prevalência nos estados da Bahia, Rio de Janeiro, Minas Gerais, Maranhão e Pernambuco. O diagnóstico laboratorial é feito pelo hemograma, teste de solubilidade para a $\mathrm{Hb}$ S, teste de falcização e pela detecção da Hemoglobina Mutante através da eletroforese de hemoglobina em $\mathrm{pH}$ alcalino ou ácido (CEHMOBMG et al., 2009).

A gestação na doença falciforme representa uma situação de risco maternofetal, independente do genótipo (SS, SC ou S-ß-Talassemia). A gestação pode agravar a doença, com piora da anemia e aumento da frequência e gravidade das crises dolorosas e das infecções, interferindo na evolução normal da gestação. Os riscos materno-fetais incluem aumento das crises vaso-oclusivas no pré e pós-parto, infecções do trato urinário, complicações pulmonares, anemia, pré-eclâmpsia e até óbito. Nas complicações fetais observam-se partos pré-termo, restrição do crescimento intrauterino devido a vaso-oclusão placentária, sofrimento fetal durante o trabalho de parto, além de elevação da taxa de mortalidade perinatal (CEHMOB-MG et al., 2009).

\section{OBJETIVOS}

\subsection{OBJETIVO GERAL}

Pesquisar as alterações fisiológicas que ocorrem em gestantes portadoras de anemia falciforme.

\subsection{OBJETIVOS ESPECÍFICOS}

Pesquisar as alterações hematológicas em gestantes portadoras de anemia falciforme.

Investigar as complicações que as mulheres portadoras de anemia falciforme possuem durante o período gestacional.

\section{METODOLOGIA}

Através de levantamento bibliográfico, mediante consulta às bases de dados Medline PubMed (US. National Library of Medicine National Institutes of Health, USA), SciELO Brazil (Scientific Eletronic Library Online) e BIREME (Biblioteca Regional de Medicina, Centro Latino-Americano e do Caribe de Informação em Ciências da Saúde) foi realizada uma pesquisa de publicações sobre as alterações fisiológicas, hematológicas que ocorrem em gestantes portadoras de anemia falciforme sem preferências de ano de publicação, porém, com prioridade para autores e trabalhos de 
grande impacto científico. Além disto, foi realizada consulta a livros de Hematologia e Saúde Pública.

\section{RESULTADOS E DISCUSSÃO}

\subsection{Fisiopatologia da doença falciforme na gravidez}

É natural que durante todo o processo gestacional ocorram mudanças anatômicas e fisiológicas resultantes da adaptação do organismo materno para a concepção de um novo indivíduo (CUNNINGHAN et al., 2000). As alterações hormonais ocorridas desde a fecundação até o processo de nidação e fixação do embrião já causam alterações no volume plasmático, devido à hipertrofia e dilatação do útero, que requer um aumento da vascularização pela necessidade de maior perfusão sanguínea (KILPATRI et al., 1999).

Na placenta, devido ao aumento progressivo, há um incremento correlato do fluxo sanguíneo uteroplacentários com a evolução da gestação, o que demanda, também, um aumento do número de vasos sanguíneos (CUNNINGHAN et al., 2000). De acordo com Souza, Filho e Ferreira (2002), o aumento do volume plasmático e da massa dos eritrócitos, que ocorre a partir da $12^{a}$ semana de gestação, leva a uma hemodiluição fisiológica conhecida como pseudoanemia da gravidez. Esse aumento da volemia plasmática é proporcional ao tamanho do feto, podendo chegar até $60 \%$ em gemelares.

O processo de hemodiluição é fisiológico e ocorre para favorecer o transporte de oxigênio como decorrência do aumento tanto do volume plasmático quanto da massa total de eritrócitos e leucócitos na circulação (CUNNINGHAN et al., 2000). Elevação do nível de eritropoetina e consequentemente dos eritrócitos são observadas após a $20^{a}$ semana de gestação. Devido à produção eritrocitária aumentada, que supera a destruição, a duração média de vida dos eritrócitos, aproximadamente 120 dias, está reduzida na segunda metade da gestação, quando a produção é marcante. Além disso, o volume corpuscular médio (VCM) do eritrócito tende a aumentar pela diminuição do seu diâmetro longitudinal e aumento da espessura de sua camada, tornando-o, também mais esférico (SOUZA; FILHO; FERREIRA, 2002).

Para as gestantes com doença falciforme, a gravidez é uma situação potencialmente grave assim como para o feto e o recém-nascido, uma vez que a microcirculação placentária é um ambiente com alto grau de desoxigenação da hemoglobina, o que pode acarretar uma maior incidência da falcização, estase e infartos placentários (BERZOLLA, 2011).

Trampont e colaboradores (2004) em um estudo histopatológico das placentas de pacientes com doença falciforme evidenciou maior incidência de fibrose das 
vilosidades, infartos e calcificações. Essas alterações, por si só, seriam suficientes para explicar as complicações típicas da gestante falciforme. A lesão da microvasculatura placentária pelos eritrócitos falcizados pode ser uma das causas da maior incidência de abortamento espontâneo e de restrição do crescimento intrauterino.

A seguir serão destacadas as principais complicações que ocorrem na gestação de pacientes portadoras de anemia falciforme:

- Infecções Urinárias: A situação de estresse do processo gestacional parece levar a uma série de complicações, sendo a infecção do trato urinário uma das mais recorrentes (HORGER; FACOG, 1971; SERJEANT, 1983; NASCIMENTO, 2000; SERJEANT et al., 2005). A heterozigose para as hemoglobinopatias (HbSC, HbAS e $\mathrm{HbAC}$ ) é situação que cursa na maioria das vezes de forma assintomática durante a vida do paciente. Porém, em relação ao sistema urinário, Serjeant (1983) comparando gestantes com traço falciforme (HbAS) e gestantes com genótipo $\mathrm{Hb}$ AA (normais) verificou maior prevalência de infecções do trato urinário e hematúria nas gestantes $A S$. Resultado semelhante já havia sido publicado por Horger (1971) o que foi também foi encontrado por Salazar (2012) e Berzolla (2011).

Murao e Ferraz (2007) verificaram também que as mulheres com genótipo AS apresentaram maior incidência de bacteriúria assintomática, cistite e pielonefrite. Com relação a homozigose para a hemoglobina $S$, esta condição aumenta o risco para as infecções urinárias, o que pode contribuir para outras complicações como o abortamento ou trabalho de parto pré-termo (HORGER; FACOG, 1971; ARMOND, 2003; SERJEANT, 2004).

- Crises dolorosas: Salazar (2012) observou que a crise dolorosa foi 39 vezes mais prevalente entre as gestantes com alguma hemoglobinopatia quando estas eram comparadas com gestantes normais. Essa alteração clínica está intimamente relacionada aos fenômenos reológicos que levam a hemodiluição com diminuição de saturação de oxigênio, o que causa a vaso oclusão e favorece a falcização e os episódios dolorosos (MURPHY, 1986). Outros pesquisadores também relatam o aumento das crises dolorosas nas gestantes portadoras de anemia falciforme, o que ressalta a importância de novas estratégias terapêuticas para melhorar a qualidade de vida destas pacientes (ARMOND, 2003; SANTOS, SURITA e PEREIRA, 2005 e ZANETTE, 2007). É importante salientar que gestantes com crises dolorosas estão associadas a altos índices de complicações obstétricas, sobretudo o trabalho de parto pré-termo, tendo ou não alguma hemoglobinopatia (CARVAJAL, et al, 2010).

- Parto Pré-maturo: Segundo Trampont e colaboradores (2004), as gestantes com anemia falciforme estão sob maior risco de desenvolver parto prematuro, sendo que $30 \%$ a $50 \%$ destas gestações evoluem para o parto antes de completar 36 semanas de gestação. A idade gestacional média na ocasião do parto é de 34 semanas nestas pacientes. Horger (1971), encontrou um percentual de $24 \%$ de 
prematuridade nas gestantes com hemoglobinopatias HbSS ou HbSC.

- Anemia: A incidência da anemia aumenta pela soma da hemodiluição própria da gravidez com a hemólise dos eritrócitos falciformes. A reposição de folato durante toda a gestação pode auxiliar no controle da anemia. Em virtude da vasooclusão decorrente da redução do fluxo sanguíneo, há uma diminuição no volume placentário, o que acarreta menor aporte de nutrientes ao feto e anormalidades na integridade da membrana placentária, causando prejuízos tanto ao feto como à gestante (CEHMOB-MG et al., 2009).

A anemia grave foi nove vezes mais prevalente nas gestações das mulheres com hemoglobinopatias quando comparadas com gestantes normais (SALAZAR, 2012) Este resultado é semelhante a outros já publicados (HORGER, 1971; HOWARD et al., 95).

A piora da anemia é relatada por Santos (2007), que aconselha acompanhamento em serviço especializado com medidas gerais que contribuam para melhorar o prognóstico materno-fetal com a prevenção e tratamento imediato da anemia megaloblástica e ferropriva. O aumento do número de transfusões sanguíneas de maneira profilática com o intuito de reduzir a incidência de complicações como abortamento e prematuridade é discutível, pois as complicações da sobrecarga de ferro durante a gestação podem levar o agravamento dos casos.

- Complicações Cardíacas: Quanto á insuficiência cardíaca, Salazar (2012) verificou que a prevalência foi 12 vezes maior entre as gestações das mulheres com hemoglobinopatias. Aumento de alterações cardiovasculares também foi relatada em outros estudos (ARMOND, 2003; HORGER, 1971; ABRAMS, 1959). Santos, Surita e Pereira (2005) relataram restrição de crescimento intrauterino em sete mulheres com hemoglobinopatias SS. Charache (1995) descreveu o baixo peso ao nascer como uma das complicações obstétricas mais comuns na gestação de pacientes com doença falciforme. Armond (2003), ao pesquisar 46 gestantes com os genótipos SS e SC, observou uma elevada frequência $(57 \%)$ de baixo peso ao nascer em recém-nascidos de mulheres com doença falciforme.

- Bolsa Rota: O tempo de bolsa rota está associado ao início do trabalho de parto prematuro, na maioria dos casos (ARMOND, 2003). Salazar (2012) relata uma prevalência maior da bolsa rota (quatro vezes maior) além da maior incidência de Préeclâmpsia (dozes vezes maior). Corroboram com estes achados os estudos de Wiston e Atroiani (1953) e Smith (1996) que encontraram frequências de 40\%, 20\% e 12\% respectivamente, de toxemia gravídica em gestantes com doença falciforme.

- Síndrome Torácica Aguda (STA): Quanto à síndrome torácica aguda (STA) a prevalência foi 12 vezes maior entre as gestações das mulheres com hemoglobinopatias. As alterações anatômicas e fisiológicas que ocorrem durante a evolução do processo gestacional, como o aumento das demandas metabólicas, a 
as vias urinárias quanto as vias respiratórias, que agrava a susceptibilidade às infecções nestes locais com exacerbação das manifestações clínicas da doença falciforme (Rust; Perry, 95; Armond, 2003). Corroboram com esses resultados Serjeantet al. (2005); Santos (2007); Nomura et al. (2009), Berzolla (2011).

- Necessidade de Parto Operatório: Em relação ao período perinatal, verificou-se que a necessidade de parto operatório foi mais associada á mulheres portadoras de hemoglobinopatias (SANTOS, 2007). Clinicamente a opção por um procedimento cirúrgico, foi praticamente o dobro (62\%) nas gestantes com HbSS quando comparadas a gestantes normais (37\%) (SANTOS, 2007). Percentuais semelhantes foram encontrados por Koshy e colaboradores (1988) e Sun (2001).

- Mortalidade fetal: Ainda que a ocorrência de mortalidade materna e perinatal tenha diminuído significativamente nas ultimas décadas (SMITH, 1996) devido aos programas de saúde pública implantados, existe um alto risco de múltiplas complicações materno-fetais associadas às hemoglobinopatias, sobretudo as SS (HOWARD et al., 1995; SUN, 2001).

O óbito fetal, segundo Salazar (2012), foi 12 vezes mais prevalente entre as gestações das mulheres com hemoglobinopatias em relação às demais. $O$ dano placentário pode explicar a probabilidade acumulada de $25 \%$ de óbito fetal no final do primeiro trimestre. Além disso, a morte fetal neste período é cerca de três vezes maior que a encontrada após a segunda metade da gestação (POWARS et al., 1986). Nascimento (2000) relata maior incidência de óbito fetal em pacientes com hemoglobinopatias HbAS. Em gestantes com anemia falciforme, Santos (2007) descreve altas taxas de óbito fetal, demonstrando que as gestações de pacientes SS possuem maiorprobabilidade de serem acometidas por complicações obstétricas.

Quanto à natimortalidade houve associação entre a presença ou não de hemoglobinopatias, sendo nove vezes mais prevalente entre as mulheres com hemoglobinopatias quando comparadas com as demais. Nascimento (2009) relata alta incidência de óbito fetal e natimortos em gestantes com hemoglobinopatia AS quando comparadas a gestantes normais. Sun (2001) ao discutir esse fenômeno justifica que a restrição de crescimento intrauterino associada aos enfartamentos placentários podem levar a natimortalidade.

Segundo Trampont e colaboradores (2004) a redução na morbidade e mortalidade feto-materna em gestantes com doença falciforme tem sido verificada por diversos autores, sendo atribuída a melhorias no cuidado geral dispensado a essas pacientes. O acompanhamento dessas doentes por equipes multidisciplinares e a adoção de medidas como a pesquisa sistemática de hemoglobinas anormais entre as gestantes em locais com incidência elevada do gene da hemoglobina S, o cuidado prénatal criterioso, a realização regular de exames ultrassonográficos para acompanhar o desenvolvimento fetal, instituição de medidas educativas para as pacientes, incluindo 
orientação nutricional, o uso regular de ácido fólico, a capacitação das equipes de saúde visando disseminar o conhecimento sobre a doença e seu manejo correto, além do acesso das pacientes ao aconselhamento genético, podem contribuir para a redução da morbidade e mortalidade materno-fetais em gestantes com a doença falciforme.

- Outras complicações: Gestantes portadoras de hemoglobina S, sobre o genótipo HbSS apresentam um elevado risco para trombose venosa cerebral, pneumonia, pielonefrite, trombose venosa profunda, infecções pós-parto e sepse. Quando analisadas as complicações intercorrentes no período gestacional, observase uma frequência elevada dos casos de toxemia gravídica, pré-eclâmpsia, deslocamento prévio da placenta, abortamentos, trabalho de parto prematuro e restrição do crescimento fetal (VILLERS et al., 2008).

\section{CONSIDERAÇÕES FINAIS}

Dentre as doenças monogênicas, as síndromes falciformes são as mais comuns e frequentes na população brasileira (VIANA et al., 2001). Aliado as complicações que estas síndromes causam aos indivíduos afetados, a gravidez é um fator complicador, tornando uma gestação nestas condições, situação de alto risco tanto para a mãe quanto para o feto. Pela pesquisa realizada fica evidente a complexidade de uma gestação em portadores das síndromes falciformes, o que justifica ainda mais a importância da prevenção para o aparecimento de novos casos através de aconselhamento genético e do maior acesso da população a programas que visam o diagnóstico das hemoglobinopatias, como os já realizados através do Teste do Pezinho.

\section{REFERÊNCIAS}

ARMOND, S. C. Complicações intercorrentes no período gestacional e resultado perinatal de pacientes portadoras de hemoglobinopatia SS e SC. Dissertação (mestrado): Universidade Federal de Minas Gerais, Belo Horizonte, 2003

BERZOLLA,C.;SELIGMAN,N.S.;AISHA,N.;DYSART,K.;BAXTER,J.K.;BALLAS,S.K. Sickle Cell Disease and Pregnancy: Does Outcome Dependo on Genotype or Phenotype. International Journal of Clinical Medicine.; 2, 313-317, 2011.

CARVAJAL,A.J.M.; PENÃ-MARTÍ,G.; CARRASCO,G.C.; MARTIN-PENÃ, A.J.. Intervenciones para el tratamiento de la crisis drepanocítica dolorosa durante el embarazo (Revision Cochrane traducida). In: Biblioteca Cochrane Plus Número 4. Oxford, 2009.. 
CEHMOB-MG et al., Manual de Acompanhamento da Gestante. Ministério da Saúde. Belo Horizonte, MG, 2009.

CHANRACHE S. Management and therapy of sickle cell disease. Maryland: National Institutes of Health, 1995.

FAILACE R. Hemograma: manual de interpretação. 3a ed. Porto Alegre, Artes Médicas Sul, 2009.

HORGER, E. Sickle cell and sickle cell-hemoglobin c disease during pregnancy. British journal of Obstetrics and Gynaecology, S.I.,1971.

HOWARD RJ et al. Pregancy in sickle cell disease in the UK: results of a multicenter survey of the effect of prophylactic blood transfusion on maternal and fetal outcome. British Journal of Obstetrics and Gynaecology. London, v.102, n.12, 1995.

LEVENO,L. et al. Maternal Child Nursing Care, Elsevier Health Sciences, 2013.

LUKENS,J.N. Hemoglobinopathies S,C,D,E and O and associated diseases. In: LEE RG, BITHELL TC, FOERSTER J, ATHENS JW, LUKENS JN. Wintrobe's Clinical Hematology. Philadelphia:Lea \& Febiger; 1993.p. 1061-1101.

MURAO, M.; FERRAZ, M. H. Traço falciforme: heterozigose para hemoglobina S. Revista brasileira de hematolologia e hemoterapia. S.I., v. 29, n.3, p. 223-225, 2007.

MURPHY, J. R. The Hemoglobin C.C. Disease: rheological properties of erythrocytes and abnormalites in cell water. Journal of Clinical Investigation. S.I., v. 47, p. 14831495, 1968.

NAOUM, P.C. Interferentes eritrocitários e ambientais na anemia falciforme. Revista Brasileira de Hematologia e Hemoterapia. São Jose do Rio Preto, v. 22, n.1, p. 5-22, 2000.

NASCIMENTO, MLP. Abortos em mulheres portadoras de hemoglobina S (AS) Revista brasileira de hematologia e hemoterapia. S.I., v. 22, n. 3, p. 424, 2000.

NOMURA, R. M. Y. et al. Resultados maternos e perinatais em gestações complicadas por doenças falciformes. Revista Brasileira de Ginecologia e Obstetricia. S.I., v. 32, n. 8, p. 405-411, 2010. 
POWARS, D. et al. Pneumococcal septicemia in children with sickle cell anemia. The Journal of the American Medical Association, Chicago, v. 245, n. 18, p. 1839-1842, 1981.

SAKAMOTO, T. M.; Hemoglobinopatias e anemias em gestantes no Hospital Universitário de Campo Grande- MS. Dissertação (Mestrado em Saúde Coletiva) Universidade Federal de Mato Grosso do Sul, Campo Grande, 2007.

SALAZAR, E. Complicações Intercorrentes nas gestações e os resultados perinatais das mulheres com hemoglobinopatias acompanhadas no serviço de gestação de alto risco, Dissertação (Mestrado em Saúde e Desenvolvimento da Região Centro-Oeste) - Universidade Federal de Mato Grosso do Sul, Campo Grande, 2012.

SANTOS, S. N.; SURITA, F. G.; PEREIRA, B. G. Resultados maternos e perinatais em portadoras de anemia falciforme. Revista de Ciências Medicas. S.I., v. 14, n. 5, p. 415419, 2005.

SERJEANT, G. R, Sickle haemoglobin and pregnancy British. Medical Journal, S.I., v. 287, n. 3, p. 628-30, 1983.

SERJEANT,G.R.; LOY, L.L.; CROWTHER,M.B.; HAMBLETON,I.R.; THAME, M. Outcome of pregnancy in homozygous sickle cell disease. Obstet Gynecol. ;103:127885,2004

SMITH, J.A. et al. Pregnancy in sickle cell Disease: Experiece of the Cooperative Study of Sickle Cell Disease. Blood, New York, v.87, n.2, 123-45, 1996.

STUART, M. J.; NAGEL, R. L. Sickle-cell disease. Lancet. v. 364, n.9442, p. 1343-60. 2004

SUN, M.P. et al. Sickle cell disease in pregnancy: Twenty years of experiece at Grady Memorial Hospital, Atlata, Georgia. American Journal of Obstetrics and Gyecology, Atlanta, v. 184, n 6, 2001.

TRAMPONT, P.; ROUDIER, M.; ANDREA,A.M. The placental-umbilical unit in sickle cell disease pregnancy: a model for studying in vivo functional adjustments for hypoxia in humans. Human Pathology. 35(11):1353-9, $2004 .$. 
VIANA, B. L. M. S et al. Prevenção de hemoglobinopatias a partir de estudo em gestantes. Revista Brasileira de Hematologia e Hemoterapia.,S.I., v. 23, n.1, p. 31$35,2001$.

VILLERS M. S. et al. Morbidity associated with sickle cell disease in pregnancy. American Journal of Obstetrics and Gynecology. S.I., v. 199, p. 121-125, 2008.

WISTON, H. G; ATROIANI, J. R. Cell Disease in Pregnancy. British Journal of Obstetrics and Gynaecology, S.I., v. 2, n.1, p.73-77, 1953.

ZANETTEAMD. Gravidez e contracepção na doença falciforme. Revista Brasileira de Hematologia e Hemoterapia, 29(3): 309-312, 2007. 